\title{
Instability studies at the CERN Proton Synchrotron during transition crossing
}

\author{
M. Migliorati* \\ University of Rome 'La Sapienza' and INFN Sez. Romal-00185 Roma-Italy \\ and CERN, 1211 Geneva, Switzerland
}

S. Aumon, E. Koukovini-Platia, A. Huschauer, E. Métral, and G. Sterbini

CERN, 1211 Geneva, Switzerland

N. Wang

Institute of High Energy Physics, 100049 Beijing, China

(Received 16 May 2018; published 13 December 2018)

\begin{abstract}
The CERN Proton Synchrotron (PS) routinely crosses transition energy at around $6 \mathrm{GeV}$ in order to accelerate protons that are injected in the Super Proton Synchrotron (SPS) or transferred to users of fixed target experiments. Depending on the beam parameters and intensity, a fast vertical coherent instability occurs during transition crossing. The instability, characterized by beam losses and a frequency spectrum in the range of 500-900 MHz, represents an important intensity limitation for the neutron time-of-flight (nTOF) beam, and in general could represent a bottleneck for future high intensity beams. In order to better understand the nature and the source of the instability and to find possible mitigations, a dedicated measurement campaign took place. Parallel to the measurements, beam dynamics simulations have been performed to study the observed instability. In particular, single bunch effects have been simulated using the PS transverse beam coupling impedance model developed over recent years. In this paper we present the measurements results along with the obtained instability thresholds. Different beam configurations and stabilizing effects, such as the gamma jump scheme and the octupole-induced tune spread, are also considered. The measurements results are compared with simulations.
\end{abstract}

DOI: 10.1103/PhysRevAccelBeams.21.120101

\section{INTRODUCTION}

The CERN Proton Synchrotron (PS) routinely crosses transition energy [1] at around $6 \mathrm{GeV}$ in order to accelerate protons that are injected in the Super Proton Synchrotron (SPS) or transferred to users of fixed target experiments. This critical passage for the accelerator is performed using a second order gamma jump scheme [2]. When the beam energy approaches the transition energy, the momentum compaction factor is modified by means of special doublet- and triplet-pulsed quadrupoles to keep the beam at a safe distance from transition. Eventually, the beam unavoidably crosses transition for a short period of time but at very high speed [3]. In Fig. 1, the schematic layout of the PS lattice is shown. In addition to the

\footnotetext{
*mauro.migliorati@uniroma1.it
}

Published by the American Physical Society under the terms of the Creative Commons Attribution 4.0 International license. Further distribution of this work must maintain attribution to the author(s) and the published article's title, journal citation, and DOI. combined function main magnets, the positions of the dedicated transition quadrupoles are marked.

Depending on the beam parameters and intensity, a vertical coherent instability occurs during transition crossing [4], producing fast beam losses. A series of studies has been performed in the past years to characterize the instability and the beam behavior under different machine settings [5-8]. In particular, the instability mechanism was investigated [9] employing beams dedicated to the neutron time-of-flight (nTOF) facility, which require high nominal beam intensity of around $800 \times 10^{10}$ protons per bunch (ppb) [10]. Generally, in order to cure this instability a longitudinal emittance blow-up is performed with a $200 \mathrm{MHz}$ cavity system at injection energy [4].

Concerning the Large Hadron Collider (LHC) beam, the instability does not occur with nominal intensity. However, due to the scheduled LHC Injectors Upgrade (LIU) project, a doubling of the beam intensity is expected. Consequently, it is important to investigate the safety margin with respect to this instability, as well as identify the actions that could raise any intensity limitation. For this reason, the most recent measurement campaign used both nTOF and LHC 


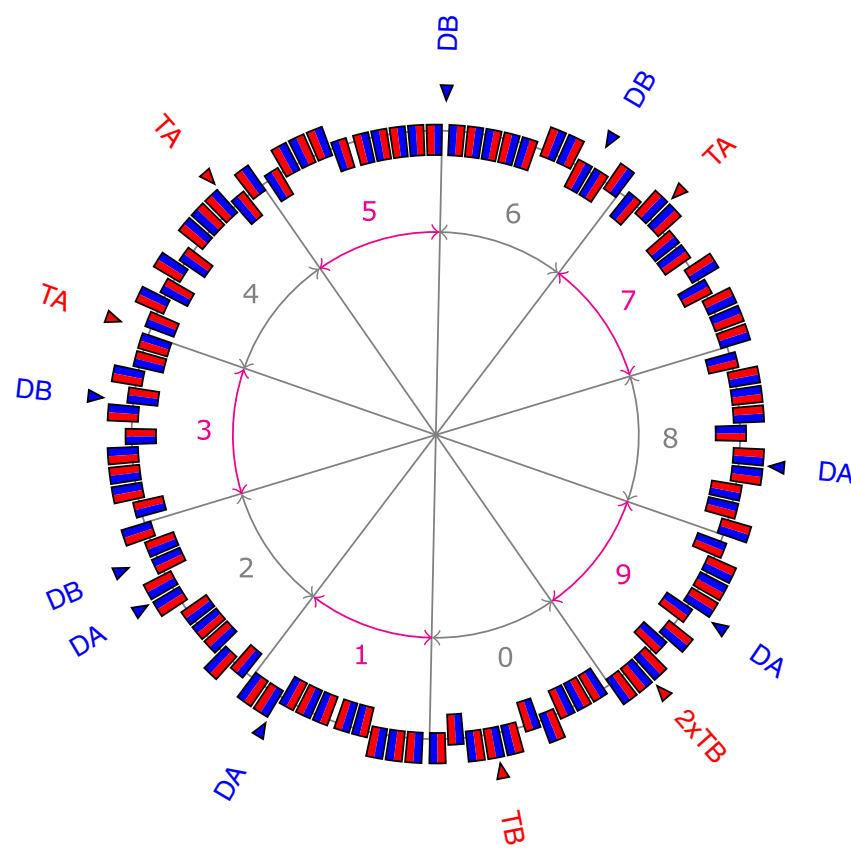

FIG. 1. Schematic layout of the PS and its 10 sectors. Blue and red elements correspond to the focusing and defocusing parts of the combined function main magnets, respectively. Furthermore, the locations of dedicated transition doublet (DA and DB) and triplet (TA and TB) quadrupoles are shown.

beams, with and without the gamma jump scheme, and the results are presented in the next section.

In parallel with the machine measurements, a new coupling impedance model of the PS has been developed in both longitudinal [11] and transverse planes [12]. The effects of the wakefields on the transverse beam dynamics have been evaluated using the PyHEADTAIL [13] macroparticle tracking code, and are discussed in Sec. III, while Sec. IV is dedicated to simulations of mitigation effects caused by machine nonlinearities. Finally, the instability mechanism is reviewed in Sec. V, before the concluding remarks.

\section{INSTABILITY MEASUREMENTS}

In order to study the transverse beam instability observed at transition energy, three different beams in terms of longitudinal emittance have been used: the nTOF beam with a nominal normalized root mean square (RMS) longitudinal emittance of $\varepsilon_{z, \mathrm{RMS}}=0.48 \mathrm{eVs}$, the LHC beam with a longitudinal emittance of $0.25 \mathrm{eVs}$, and the LHCINDIV (LHC Individual Bunch Physics Beam) characterized by a smaller longitudinal emittance that can reach values as low as $0.05 \mathrm{eVs}$. Each beam requires a different machine configuration and it is possible to vary some characteristics, for example the emittance, within certain limits. The beams are injected with a kinetic energy of $1.4 \mathrm{GeV}$ from the PS Booster (PSB), and accelerated at different harmonic numbers $h$ of the revolution frequency,

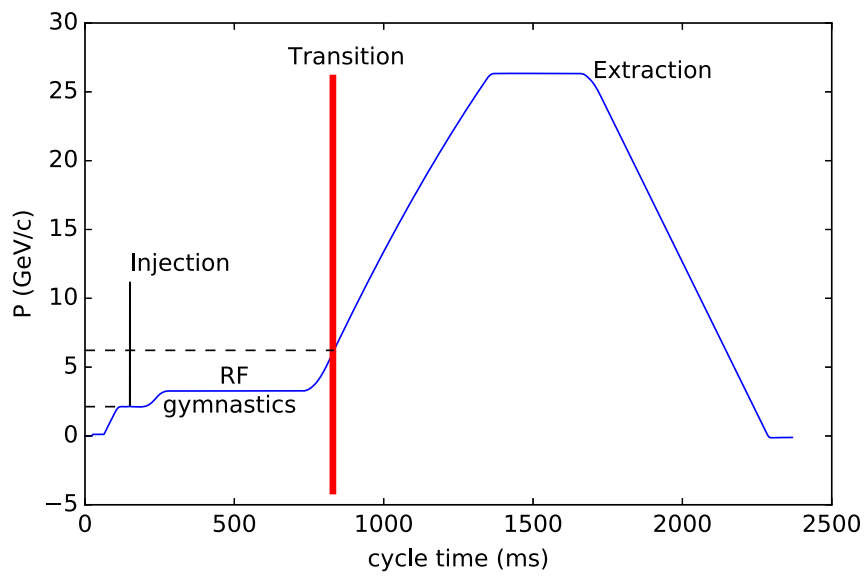

FIG. 2. Typical PS magnetic cycle of LHCINDIV beams.

depending on the bunch type: $h=8$ for nTOF, $h=21$ for LHC, and $h=16$ for LHCINDIV. As a consequence, different $\mathrm{rf}$ gymnastics have to be performed before acceleration. As an example, in Fig. 2, the PS magnetic cycle for the LHCINDIV beam is illustrated. However, in some cases, a dedicated cycle has been set up and beams with different longitudinal emittances could be accelerated.

In Table I, the main parameters used in measurements and simulations are summarized: the root mean square longitudinal emittance $\varepsilon_{z, \text { RMS }}$, the relative momentum spread $\Delta P / P_{0}$, and the total bunch length, which is approximately 4 times the RMS bunch length. These numbers, relative to the PS before acceleration, have to be considered as average values since they can vary during the measurements, and, for some machine development sessions (MDs) they have been changed deliberately. An example of the measured longitudinal phase space distribution [14] at injection for the LHC beam is shown in Fig. 3.

When the bunch intensity reaches a certain threshold for a given longitudinal emittance, a fast instability in the vertical plane arises. The instability can be observed using the vertical signal coming from a wall current monitor (WCM), a device which intercepts the wall current by means of coaxial transmission lines, distinguishing between top, bottom, left and right signals. These signals can be summed or subtracted. The sum signal corresponds to the bunch profile, and the difference signal gives indication on the transverse displacements along the bunch (being zero if the bunch passes in the center of the WCM).

TABLE I. Typical beam parameters used during measurements for nTOF, LHC, and LHCINDIV beams before acceleration.

\begin{tabular}{lccc}
\hline \hline Beam & $\varepsilon_{z, \text { RMS }}(\mathrm{eVs})$ & $\Delta P / P_{0}\left(\times 10^{-3}\right)$ & Duration $(\mathrm{ns})$ \\
\hline nTOF & 0.48 & 2.5 & 140 \\
LHC & 0.25 & 1.5 & 110 \\
LHCINDIV & 0.05 & 0.8 & 20 \\
\hline \hline
\end{tabular}




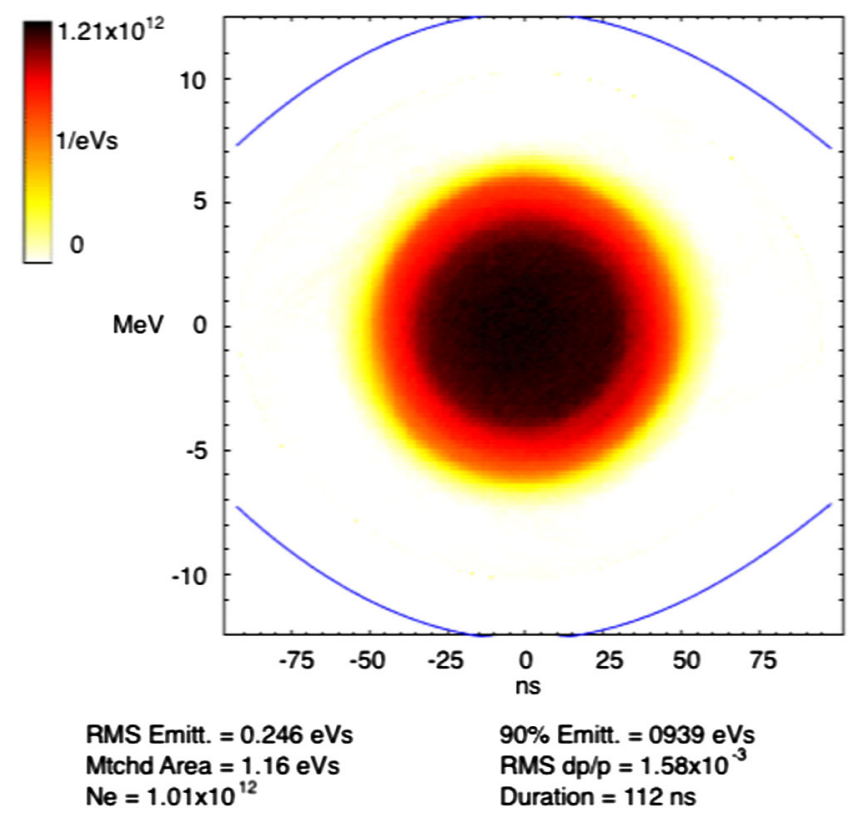

FIG. 3. Reconstruction of a typical longitudinal phase space distribution of the LHC bunch.

In Fig. 4 the measured sum and difference horizontal and vertical signals for the nTOF beam are shown. The left plot corresponds to a beam intensity just above the instability threshold, whereas the right plot to a higher intensity. The head of the bunch is on the right side of each plot. For a stable bunch, below the threshold, the sum and difference signals are very similar to those on the left side of the figure, except that no oscillation is visible in the difference signal. In both cases, the signals were recorded at the same time along the cycle, namely just before transition crossing. As we can see from the figure on the right, the highest intensity causes particle losses and an emptying of the center of the bunch where the longitudinal density is higher and the instability stronger. Saturation is also observed on the difference vertical signal due to the strong instability. The horizontal signal, instead, is essentially not influenced. A small perturbation in the case of strong instability can be observed, possibly due to coupling of the horizontal and vertical planes. As the beam dynamics evolves in the machine, the vertical difference signal behaves as a traveling wave. Particles at the center of the bunch start to be lost also in the case of a weaker instability (left side of the figure) at later times. In both cases, the overall result is a reduction of the beam current due to particles hitting the vacuum chamber.

Typical signals as those represented in Fig. 4 are a clear sign of the instability. It is also possible to determine the instability threshold by monitoring, with a beam current transformer, the beam losses during transition crossing, as shown in Fig. 5. The beam current after transition is represented as a function of current before transition for various longitudinal emittances. Without instability, the points should be aligned with the dashed line $y=x$. All the measurements refer to cases without gamma jump.

A deeper analysis of the instability can be done by considering the spectrum of the WCM difference signal in a given time interval during transition crossing. Such a spectrogram can be used to investigate the source of the instability. On the left side of Fig. 6, the bunch population obtained with the beam current transformer versus the PS cycle time is illustrated. The right plot shows the spectrogram from $0.2 \mathrm{GHz}$ up to $1.5 \mathrm{GHz}$ during $2.5 \mathrm{~ms}$ at transition crossing obtained by the Fourier transform of the difference signal of the WCM for the case corresponding to the instability shown in Fig. 4 (left side).

As we can see from the figure, the spectrum of the WCM difference signal has a maximum in a frequency range between $500 \mathrm{MHz}$ and $900 \mathrm{MHz}$. As we will see later, this information is useful to identify the possible sources of
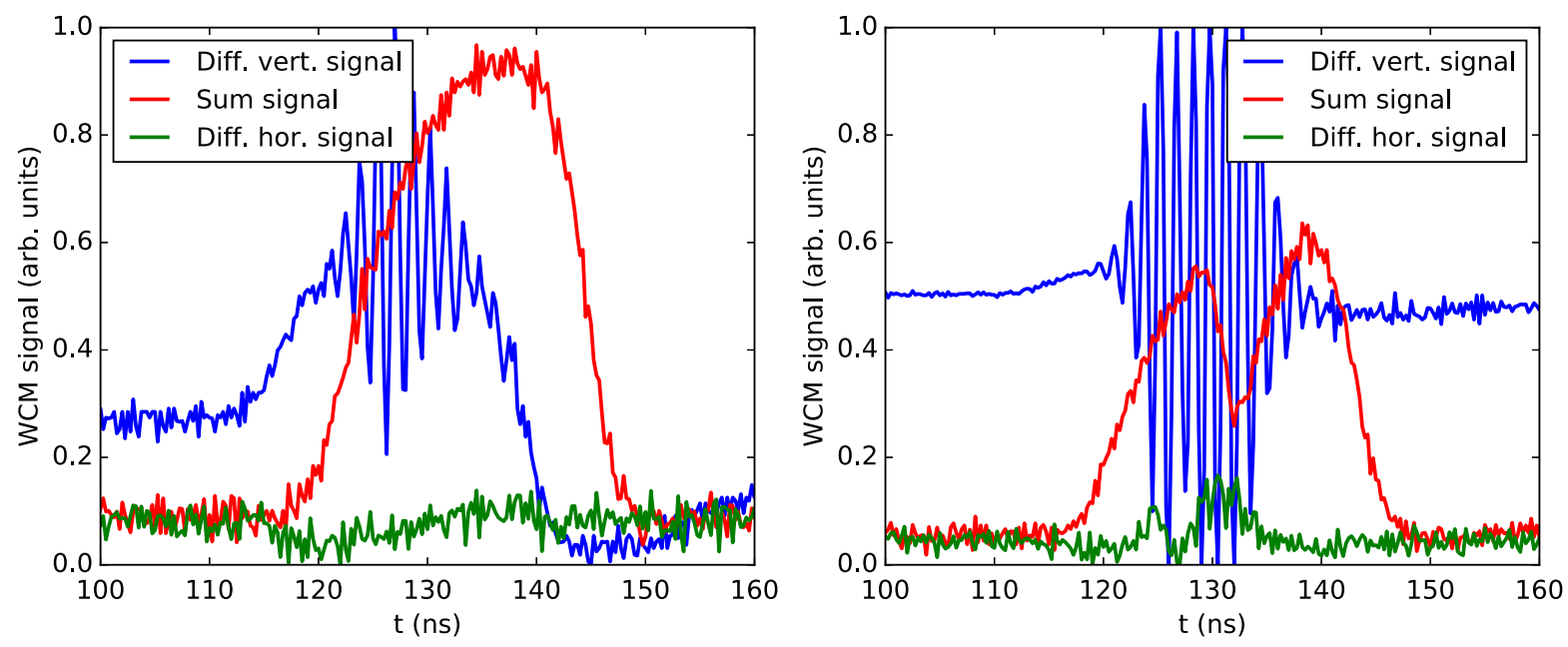

FIG. 4. Sum and difference signals from a wall current monitor observed during instability. Left side weak instability, right side strong instability. The head of the bunch is on the right side of each plot. 


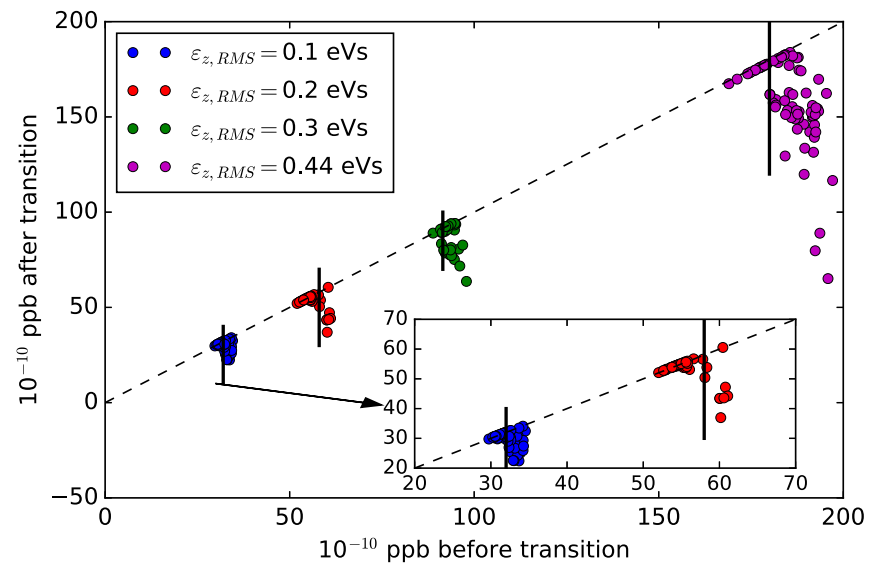

FIG. 5. Bunch population after transition as a function of bunch population before transition for $\varepsilon_{z, \mathrm{RMS}}$ of $0.1,0.2,0.3$, and $0.44 \mathrm{eVs}$. instability. In addition to that, the spectrum can be also used to evaluate the growth rate of the instability [7], which gives an indication of the time during which it develops. This time depends on the bunch intensity, and it is in the order of 0.1-1 ms, corresponding to about 50-500 turns.

An important observation concerning the spectrum is that, while for the nTOF beam the spectrogram shows a clear evidence of the excited frequencies, which allows us to identify the frequency range of the instability, this does not happen for very short bunches, such as LHCINDIV. In Fig. 7 we show an example for two different longitudinal emittances: $0.033 \mathrm{eVs}$ (left side) and $0.076 \mathrm{eVs}$ (right side).

The explanation of this difference is the following: as the bunch gets shorter, few oscillations occur within the bunch and the Fourier transform cannot be accurate any more. However, the difference signal in the time domain of the LHCINDIV beams, shown in blue and green in Fig. 8, demonstrates similar characteristics as the one of the nTOF beam, shown in red. This is a clear indication that the
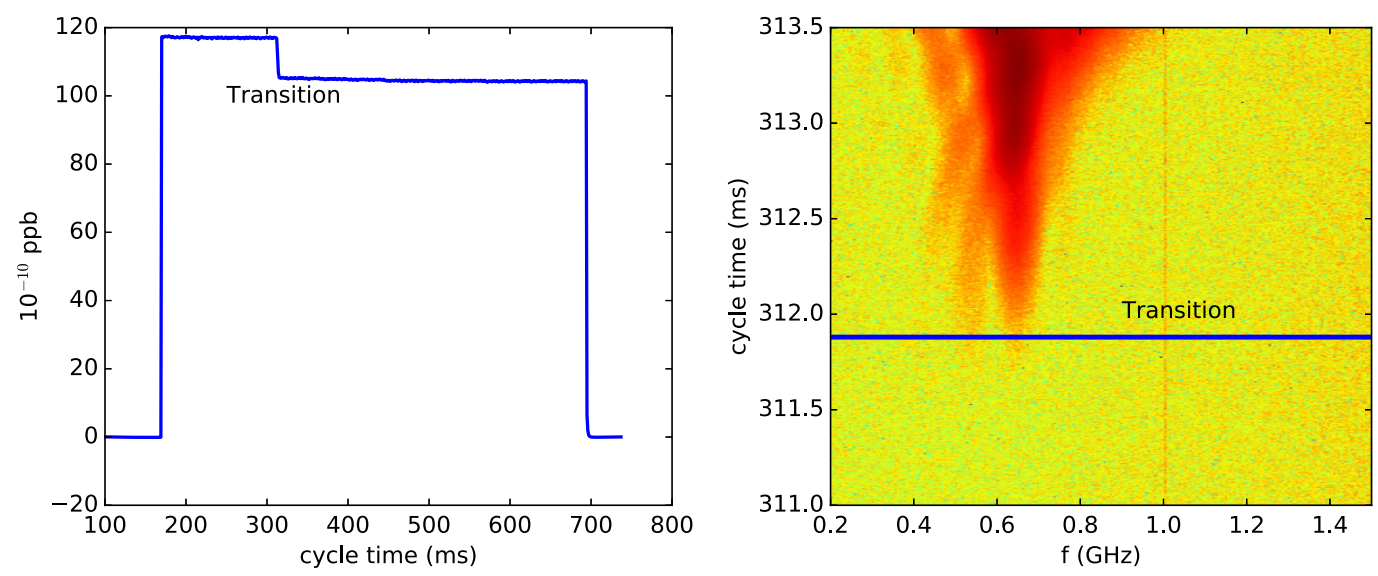

FIG. 6. Bunch population versus time during a complete PS cycle obtained with a beam current transformer (left) and spectrogram during transition crossing obtained by the Fourier transform of the difference signal of the WCM (right). Both cases correspond to the instability on the left side of Fig. 4.
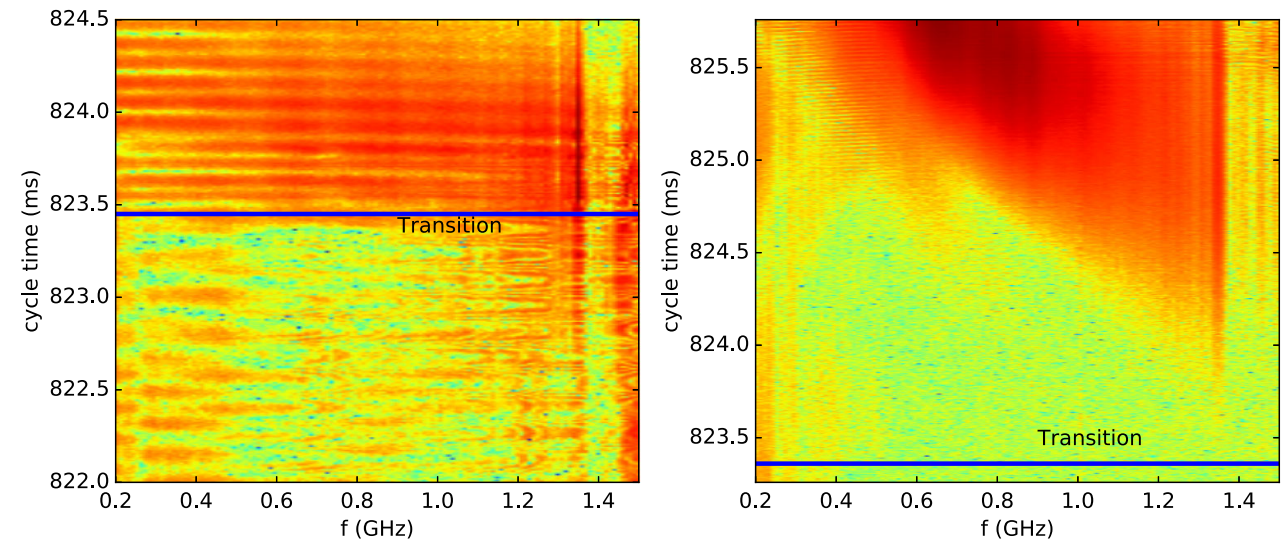

FIG. 7. Spectrograms during transition crossing obtained by the Fourier transform of the difference signal of the WCM for an LHCINDIV beam with longitudinal emittance of $0.033 \mathrm{eVs}$ (left side) and $0.076 \mathrm{eVs}$ (right side). 


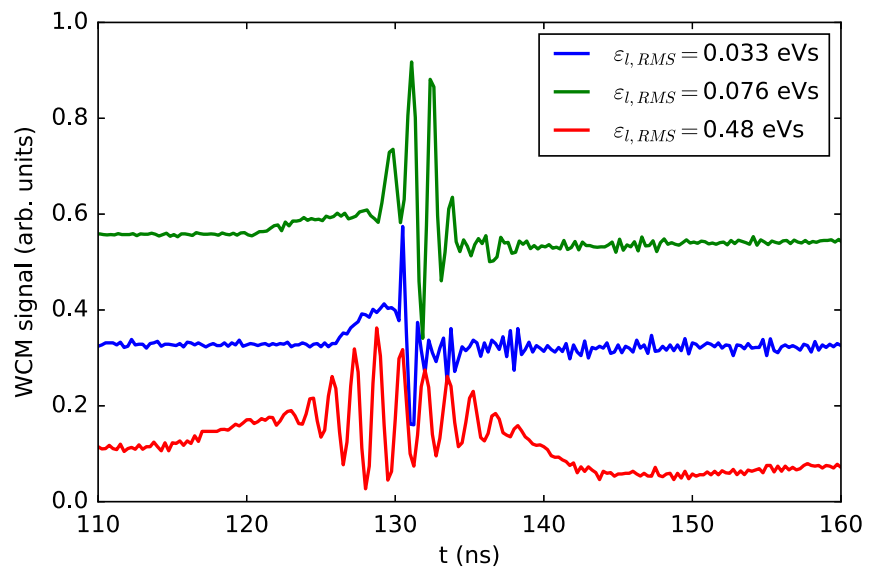

FIG. 8. Vertical difference signals from a wall current monitor observed during instability with three different longitudinal emittances.

observed instability is caused by the same source, and it has the same frequency range.

One of the main reasons for the study of the instability at transition crossing is related to the LIU project. It is important to determine the instability threshold for the LHC beam including the gamma jump in order to ensure that the PS can successfully deliver LHC beams to the SPS with intensity of about $26-27 \times 10^{10} \mathrm{ppb}$. Thanks to an intense rf gymnastics, the 6 bunches injected in the PS are first subjected to a triple splitting, allowing us to accelerate 18 bunches that cross the transition energy. At top energy, the bunches go through two double splittings [15], giving 72 bunches at extraction. Therefore, transition is crossed with a single bunch intensity four times higher than extraction. MDs have been dedicated to find the instability threshold for LHC beams, and the results are shown in Fig. 9. The measurements indicate that the threshold is around $200 \times 10^{10} \mathrm{ppb}$, giving a safety margin of about a

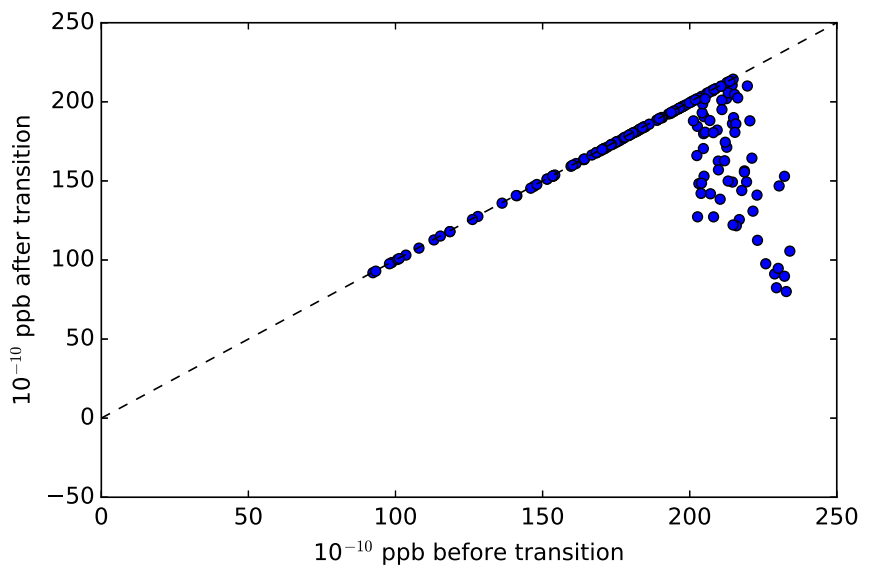

FIG. 9. Bunch population before transition as a function of bunch population after transition for the LHC beam with gamma jump. factor 2 with respect to $4 \times\left(26 \times 10^{10}\right) \mathrm{ppb}$. This margin can most likely be increased by a further optimization of the cycle settings.

\section{IMPEDANCE MODEL AND PYHEADTAIL SIMULATIONS}

A transverse coupling impedance model [16] of the PS machine has been developed in Ref. [12] including the most significant impedance sources. The model has been evaluated at different energies in order to take into account the indirect space charge contribution. In the same reference, the reactive effective impedance was compared with the measured tune shift, and the conclusion was that about 5\%-30\% of the imaginary impedance was missing. One important point about the simulations of the instability at transition described here is that they allow also to check the real part of the impedance model, and in particular its dependence with frequency.

The total vertical impedance, i.e., the sum of the dipolar and quadrupolar components, is shown in Fig. 10 up to $1.5 \mathrm{GHz}$ at a total energy of about $7 \mathrm{GeV}$. In the same plot, the kickers' impedance is shown in dashed lines. As we can see, the real part of the impedance has a broad band behavior in the same frequency range of the observed instability at transition, and the kickers are the main source of this impedance, indicating that they are the principal cause of the observed instability. Moreover, in Ref. [12], a table of the effective impedance of the kickers shows that the most significant contribution is due to the kicker located in straight section 71 (KFA71). The same conclusion is also confirmed by the impedance localization measurement technique of the same paper. The narrower peaks at higher frequencies are due to other elements. Transition steps are responsible for the peak observed at $1.23 \mathrm{GHz}$, while the peak at $1.4 \mathrm{GHz}$ is due to the vacuum ports. Valves and

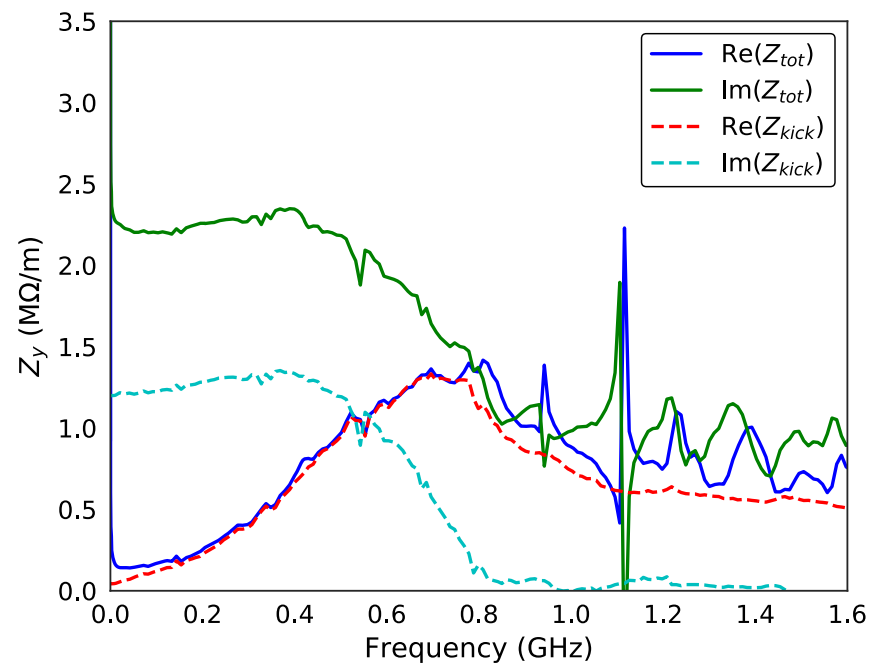

FIG. 10. Vertical impedance model of the PS machine versus frequency at $7 \mathrm{GeV}$. 
flanges are causing the narrow peaks observed at $1.1 \mathrm{GHz}$ and $0.94 \mathrm{GHz}$. Concerning the imaginary part of the impedance, the main difference between the total impedance and the contribution of the kickers is due to the indirect space charge.

This vertical impedance model has been used in the PyHEADTAIL macroparticle tracking code. For more realistic simulations, we have also included the horizontal impedance, even if we have observed that this does not change the results. The single bunch beam dynamics has then been simulated during acceleration and transition crossing under the effects of the total transverse wakefields, accounting separately for the dipolar and the quadrupolar (detuning) effects, and with the approximation of a constant indirect space charge contribution. Since we are interested in the transverse beam dynamics, and in order to simplify the analysis of the results, we ignored the longitudinal wakefields.

In order to reduce the computational time, simulations do not start from the injection energy with $\gamma=2.5$, but from $\gamma=4$. This is still sufficiently far away from transition at $\gamma=6.1$, allowing sufficient time for a potential instability to develop. The simulated bunch is in equilibrium with the rf accelerating bucket, it has a 2D Gaussian transverse distribution, and its momentum increment per turn has been defined considering the magnetic field ramp rate. As an example, the simulated bunch length and vertical normalized emittance as a function of time are shown on the left and right side of Fig. 11 for the LHC and nTOF bunches. The corresponding intensities are $27 \times 10^{10}$ for the LHC and $51 \times 10^{10} \mathrm{ppb}$ for the nTOF beams. In the same figure, on the right axis, the relativistic gamma is indicated in dashed lines.

The minimum bunch length occurs at transition crossing, while a sudden increase of the vertical emittance is observed at the same time. This sudden increase, which takes place in less than a millisecond, occurs above a given intensity threshold marking the onset of a vertical instability.
For the PyHEADTAIL simulations the bunch is divided into slices, and the vertical position of the center of mass of each slice is evaluated and weighted with the number of macroparticles in the slice. These data, computed at each turn, produce a signal that can be compared with the measured difference signal of the WCM. Indeed, very good agreement is found comparing the signal obtained with measurements and simulations. The results are shown in Fig. 12, where on the left side the two signals for the nTOF beam are represented. By adding a vertical aperture in PyHEADTAIL, the beam losses in the central part of the distribution can also be reproduced. On the right side of the figure, the sum signal of the WCM is compared with the simulated longitudinal distribution when considering a $35 \mathrm{~mm}$ vertical aperture equal to the vertical semiaxis of the elliptic beam pipe. Also in this case the similarity is excellent. Although the beam intensities are different in the figure, the goal of these comparisons is to highlight that the instability observed with PyHEADTAIL, given the current PS impedance model, has the same characteristics as the measured one.

The spectrogram of the weighted position of the vertical center of mass of the slices, turn after turn, can also be evaluated in PyHEADTAIL. The obtained signal is shown on the left side of Fig. 13 for the nTOF beam. The longitudinal emittance is $0.5 \mathrm{eVs}$. The spectrogram shows that the instability has a frequency range between $450 \mathrm{MHz}$ and $1 \mathrm{GHz}$, with the strongest part between $500 \mathrm{MHz}$ and $900 \mathrm{MHz}$, similar to the measured spectrogram shown in Fig. 6. The transverse impedance model reproduces very well the measured frequency range. Similarly to what has been observed in the measurements, also for simulations the spectrograms of smaller longitudinal emittances cannot be accurate any more due to the few oscillations occurring within the bunch, and a very large spectrum is found, not very different from that of LHCINDIV shown in Fig. 7. All these observations allow us to identify the main source of instability in the real part of the impedance of the kickers.

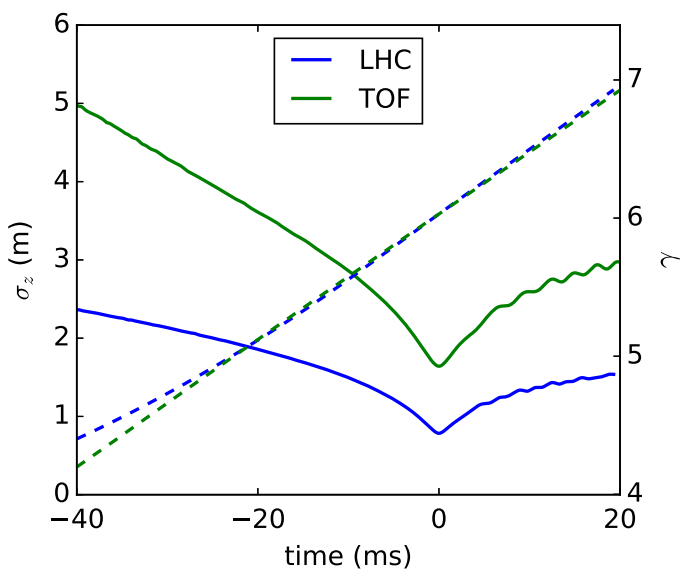

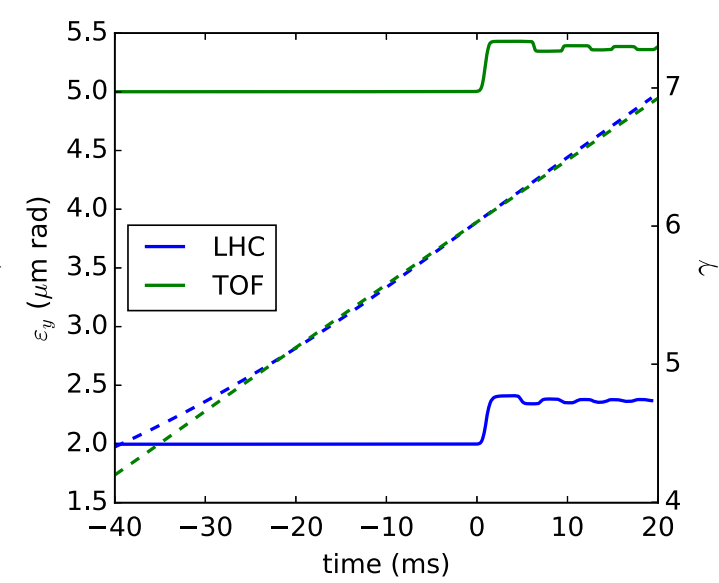

FIG. 11. Simulated bunch length (left) and vertical normalized emittance (right) as a function of time for LHC and nTOF bunches. The relativistic gamma is shown in dashed lines. The time $t=0$ has been set at transition. 

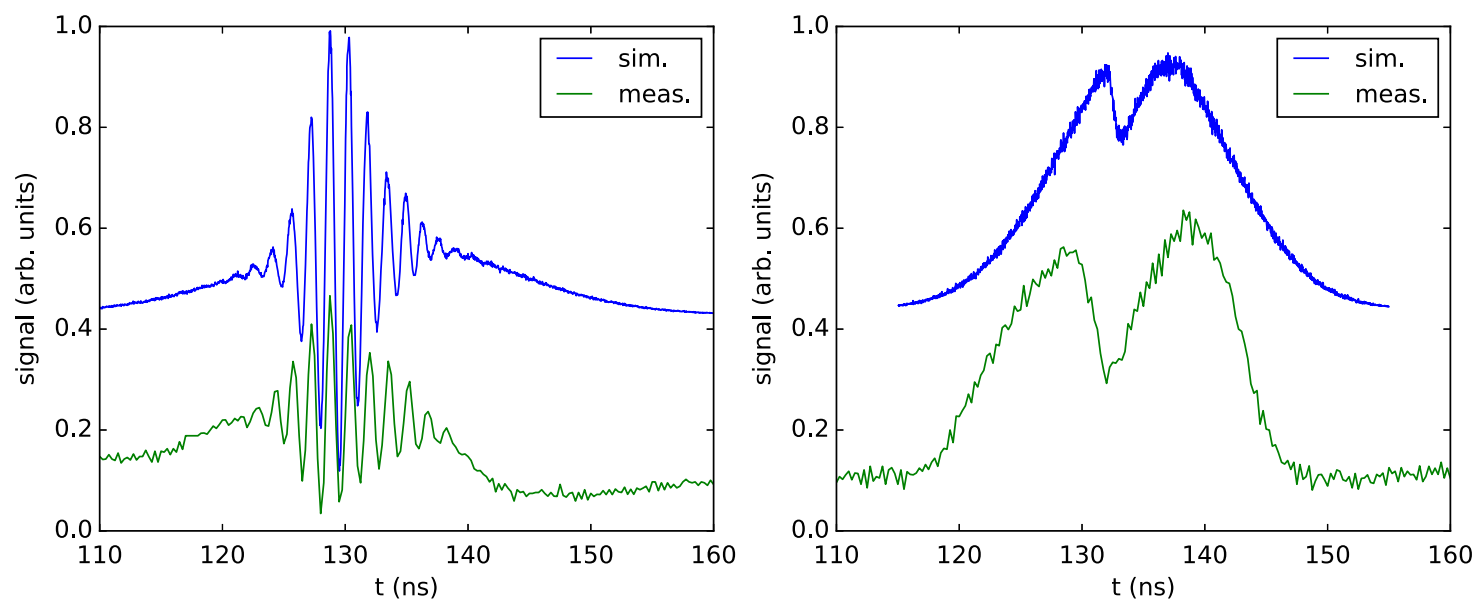

FIG. 12. Comparison between measurements and simulations for the nTOF beam. Left side: comparison between the simulated weighted position of the vertical center of mass of the slices and the WCM difference signal. The simulation results were scaled vertically and translated horizontally for comparison with the measurements. Right side: longitudinal beam distributions for the nTOF beam.

The criterion used to determine the instability threshold in simulations is a $20 \%$ increase of the normalized emittance with respect to the initial one, accompanied by macroparticle losses in the aperture used in PyHEADTAIL. The average beam pipe half-aperture of $35 \mathrm{~mm}$ is used in the simulations. For the nTOF beam, a 20\% increase of the emittance in the machine corresponds also to the beginning of the losses. A series of simulations was performed for various longitudinal emittances, and the resulting thresholds, using linear optics, zero chromaticity, and without the direct space charge effect, are represented in red on the right side of Fig. 13. In the same figure, in blue, the results of systematic measurements show a quite good agreement only for low longitudinal emittances up to about $0.15-0.2 \mathrm{eVs}$. For larger values, simulations predict stronger instabilities than measurements.
The results of these simulations, taking into account only the wakefields without any damping mechanism, are however important since they allow us to conclude that: (i) the real part of the impedance model, which is the responsible of the instability, has a frequency spectrum which agrees with the measured frequency range; (ii) the main contribution to the real part of the impedance is that of the kickers, which have been identified as the most important source of the instability. In case an increase in intensity is required in the future by the users, a study to reduce the kickers impedance might be necessary; (iii) for larger emittance values, simulations predict stronger instabilities than the measurements. This suggests that, differently from the imaginary part, the real part of the impedance model is not missing any important contribution, and, in the worst case, simulations give conservative results.
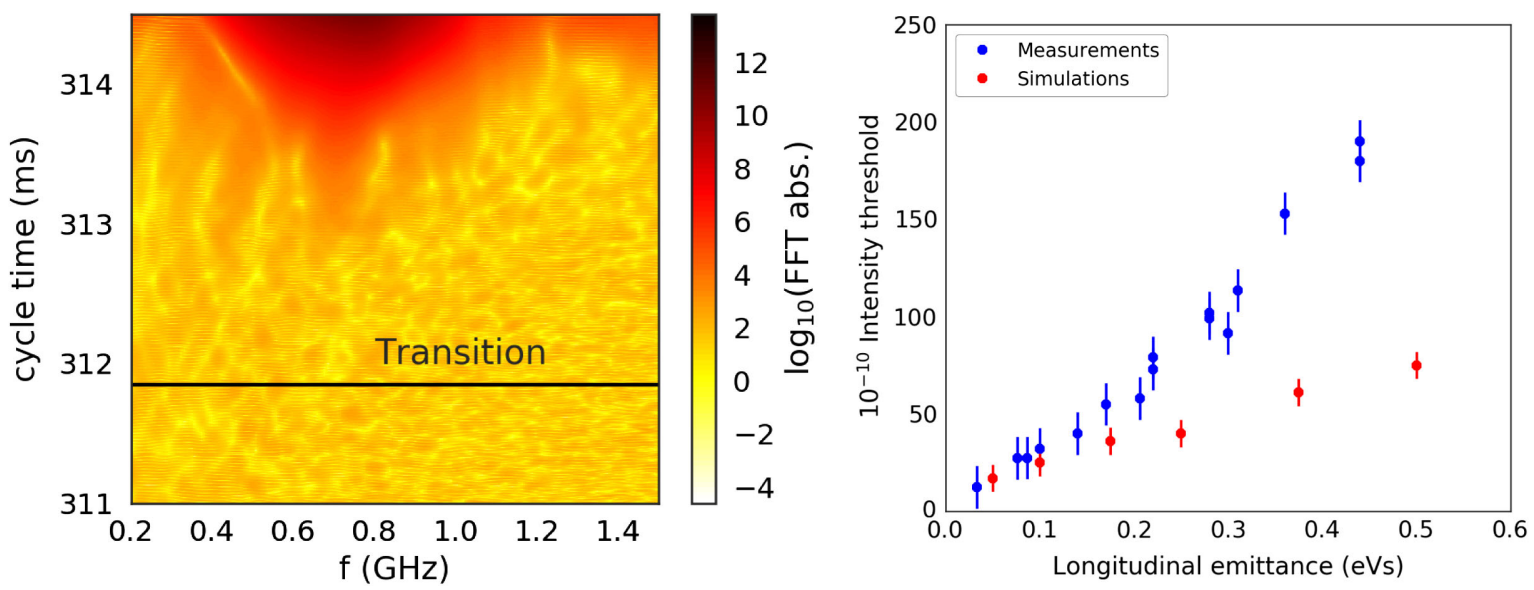

FIG. 13. Simulated spectrogram during transition crossing obtained by the Fourier transform of the weighted position of the vertical center of mass of the slices for a nTOF beam with longitudinal emittance of $0.5 \mathrm{eVs}$ (left side), and instability threshold as a function of the longitudinal emittance obtained with simulation (in red) and measurements (in blue). 
In the next section we introduce in PyHEADTAIL various damping mechanisms. The latter can increase the instability threshold with respect to the case of simulations accounting only the effect of wakefields.

\section{MECHANISMS OF INSTABILITY MITIGATION}

The main simplification of the model used so far in PyHEADTAIL, is the lack of any mechanism which in the real machine could mitigate the instability. The effects of first and second order chromaticity, direct space charge and amplitude detuning are studied in this section.

A transverse tune spread, induced for example by chromatic effects, can strongly influence the predicted threshold. First step is to introduce in simulations the first order chromaticity, defined as

$$
Q_{y}{ }^{\prime}=\left.\frac{\partial Q_{y}}{\partial \delta}\right|_{\delta=0},
$$

where $\delta=\Delta P / P_{0}$ and $Q_{y}$ is the betatron tune. By using the measured values of $Q_{y}^{\prime}$ for the nTOF cycle around transition crossing, we obtain the cyan points on the left side of Fig. 14. Below transition a negative value of $Q_{y}^{\prime}=-0.8$ was used while, above transition a positive value of 0.2 . Good agreement is found with measurements up to a longitudinal emittance of about $0.3 \mathrm{eVs}$, thus covering also the LHC beam case. Above this value of emittance the thresholds predicted by PyHEADTAIL do not increase linearly any more.

Another source of mitigation could be due to the tune spread induced by the direct space charge contribution. The direct space charge suite available in PyHEADTAIL for

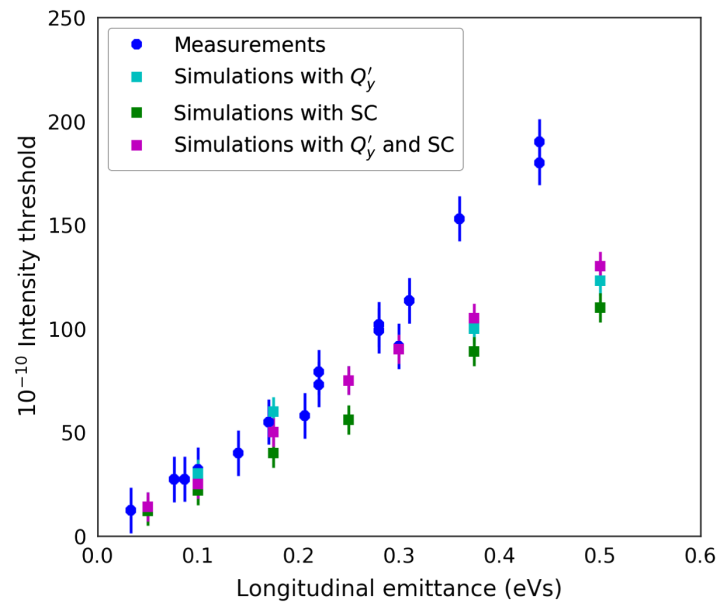

Graphics Processing Units (GPU) was used thanks to recent developments [17]. In the transverse plane, a semianalytical Bassetti-Erskine model is used [18], as well as 2D particle-in-cell solvers. The simulation results are also shown on the left side of Fig. 14 in green. As expected, the induced tune spread increases the instability thresholds compared to the case without space charge of Fig. 13.

However, by comparing the effect of space charge with respect to that of chromaticity, we observe that the latter contributes more significantly. If both effects are considered, we obtain the magenta points, showing that the two effects do not add up independently. We find a good agreement for longitudinal emittances up to slightly above $0.3 \mathrm{eVs}$, and a difference in the range of $30 \%-35 \%$ is found only for the nTOF beam, with simulations being more conservative.

On the right side of the same figure we have further investigated other existing tune spread mechanisms. The effect of the second order chromaticity has been included in PyHEADTAIL, defined as

$$
Q_{y}^{\prime \prime}=\left.\frac{1}{2} \frac{\partial^{2} Q_{y}}{\partial \delta^{2}}\right|_{\delta=0}
$$

As shown in black on the right side of Fig. 14, if we consider a value of $Q_{y}^{\prime \prime}$ of about -300, we obtain instability thresholds as a function of the longitudinal emittance very similar to the measured ones for all values of longitudinal emittance. However, measurements of this quantity performed in recent MDs indicate that around transition we expect a value of $Q_{y}^{\prime \prime}$ of about \pm 50 .

Another important mechanism that could affect the instability threshold is due to machine nonlinearities,

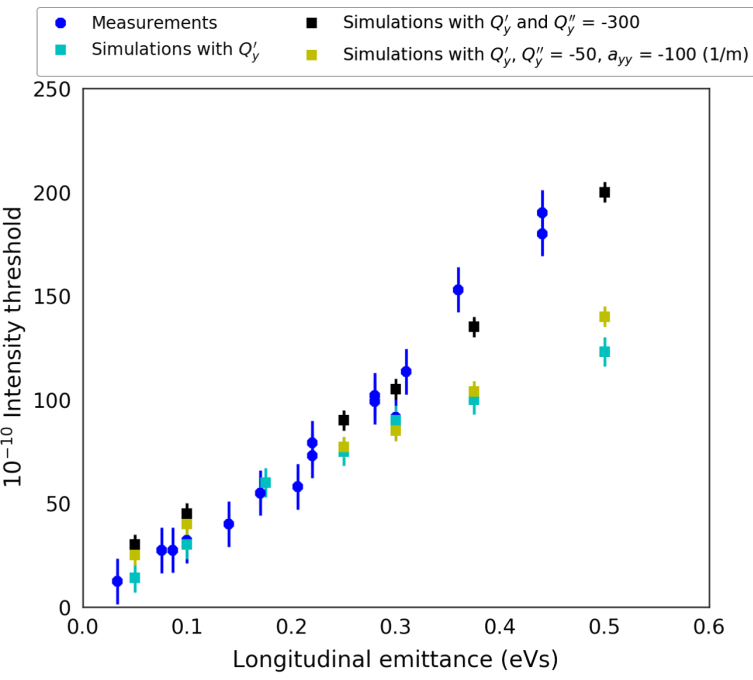

FIG. 14. Instability threshold as a function of the longitudinal emittance obtained with PyHEADTAIL. Left side: simulations with first order chromaticity (in cyan), with direct space charge (in green), and with both effects (in magenta), compared with measurements (in blue). Right side: simulations with first order chromaticity (in cyan), with a first and second order chromaticity $Q_{y}^{\prime \prime}=-300$ (in black), and with $Q_{y}^{\prime}, Q_{y}^{\prime \prime}=-50$ and an amplitude tune dependency $a_{y y}=-100 \mathrm{~m}^{-1}$ (in yellow), compared with measurements (in blue). 

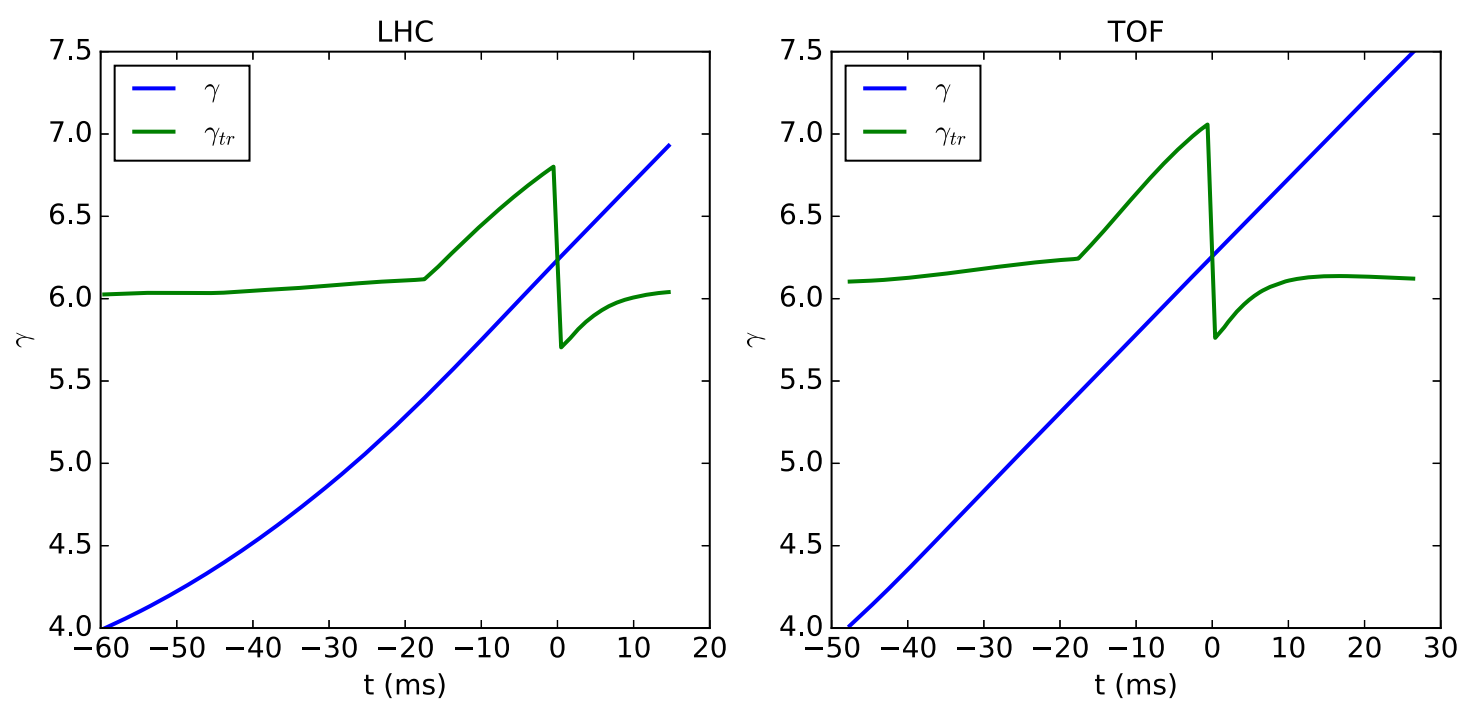

FIG. 15. Transition gamma and relativistic gamma of the beam as a function of cycle time for the LHC beam (left side) and for the nTOF beam (right side).

which can introduce an amplitude dependent tune. In PyHEADTAIL, the tune shift as a function of oscillation amplitude is defined as

$$
\Delta Q_{y}=a_{y y} J_{y},
$$

with $a_{y y}$ being the anharmonicity value and $J_{y}$ the action variable in meter. A value of $a_{y y}$ of about $-100 \mathrm{~m}^{-1}$, is in line with measurements performed in the past, and including this effect in simulations we obtain the points in yellow on the right side of Fig. 14. As in the previous cases, there is good agreement up to a longitudinal emittance of about $0.35 \mathrm{eVs}$, and for larger emittances a kind of saturation seems to appear, predicting at $0.5 \mathrm{eVs}$ a lower threshold of about $30 \%$.

It is interesting to observe that in all cases, low longitudinal emittances are almost not influenced by these effects and are in good agreement with measurements.

For the daily operation of the PS, a key feature that allows increasing the beam intensity significantly is to maintain the bunch as far as possible from transition, and then cross transition energy rapidly. This is realized with the gamma jump scheme routinely used in the PS, which can also help to reduce the longitudinal mismatch due to space charge and the inductive part of the longitudinal impedance [2].

The gamma jump scheme in the PS can vary depending on the way the special doublet and triplet pulsed quadrupoles are programmed, but in any case the instability threshold is pushed to higher intensities. Indeed, from the measurements presented in Sec. II, we observe an intensity increase for the LHC beams from $80 \times 10^{10} \mathrm{ppb}$ without gamma jump to, at least, $200 \times 10^{10} \mathrm{ppb}$ with gamma jump. This allows us to increase the beam intensity by a factor 2.5. A slightly better result is obtained with the nTOF beam, where the threshold increases from
$200 \times 10^{10} \mathrm{ppb}$ to $830 \times 10^{10} \mathrm{ppb}$ without and with gamma jump respectively, giving a gain factor of about 4. In Fig. 15, the transition gamma as a function of time obtained from the optics model of the machine for the LHC (left) and nTOF beam (right) is shown. The relativistic gamma is illustrated in the same plot.

The same gamma jump schemes have been included in PyHEADTAIL. For the nTOF beam this leads to a three times higher threshold compared with simulations without the jump. In the case of the LHC beams, the ratio of the thresholds with and without gamma jump is about 5.2.

In addition to the well-known gamma jump scheme, the aforementioned simulations showed that several sources of transverse tune spread can help mitigate the instability. For example, the importance of an amplitude dependent detuning was tested in the machine by powering the octupoles and measuring the threshold's dependence on the octupole current. As shown in Fig. 16, the octupoles can

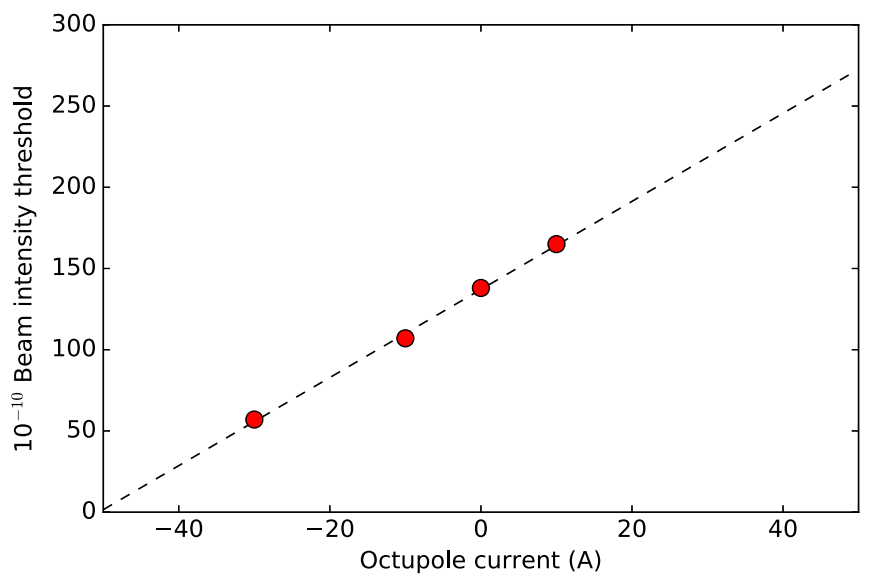

FIG. 16. Beam intensity threshold as a function of the octupole current measured in the PS. 
increase or reduce the tune spread, affecting the threshold in a positive or negative way. A linear dependence of the threshold on the octupole current is observed. This same linear behavior has been found in [19]. In the notation used in the figure, positive octupole current means negative gradient and positive vertical tune shift versus amplitude [20], which shifts the tunes of the particles to higher values, in particular at the head and tail of the bunch, increasing the bunch tune spread. The opposite happens for negative currents.

\section{REVIEW OF THE INSTABILITY MECHANISM}

In this section we review the mechanism that produces such an instability. In Ref. [4], the authors state that, when the bunch is approaching the transition energy, the longitudinal motion of the particles is almost frozen and the beam dynamics is more similar to that of a bunch in a linear accelerator rather than in a circular machine. As a consequence of this, it seems more appropriate to describe the

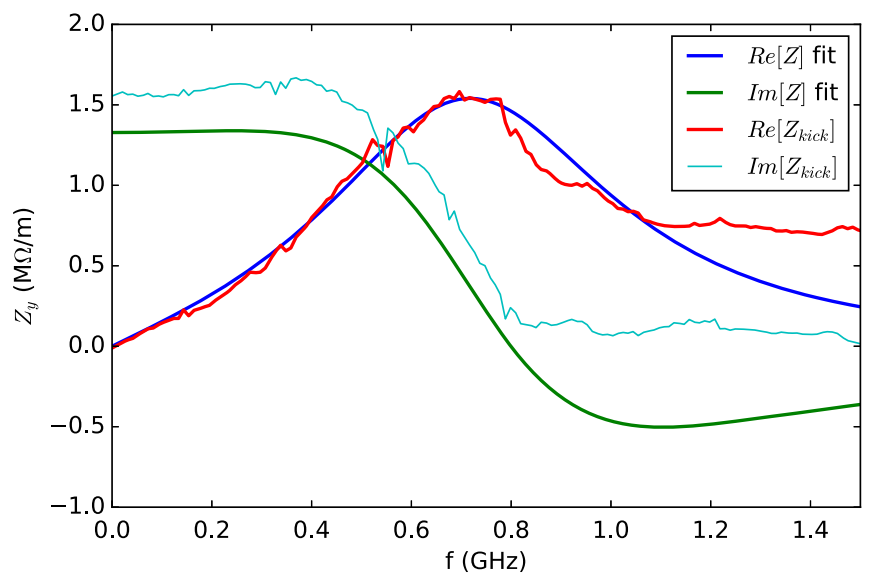

FIG. 17. Real and imaginary part of a resonator impedance used for BBU simulations.

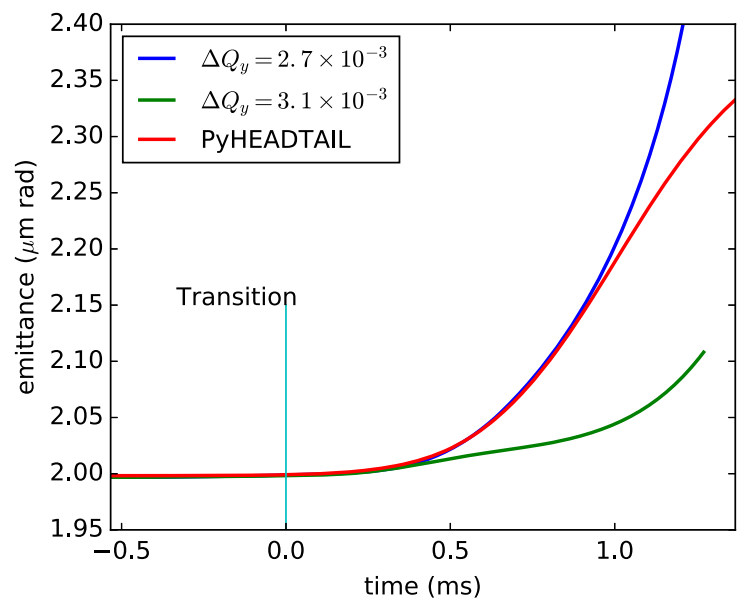

instability in terms of single bunch beam break-up [21] (BBU), with the synchrotron motion helping in alleviating the instability.

In order to better understand if the observed instability is of BBU kind, a simple simulation code has been developed. The code is based on the same approach as MuSiC [22] and does not use slices for the wakefield effects, as in PyHEADTAIL. It approximates the impedance with resonators, and it transports the wakefield from one particle to another using a matrix formalism. Unlike MuSiC, which deals with the longitudinal beam dynamics, the matrix has been developed for the transverse wakefield. More details on the code and the wakefield transport by matrix formalism can be found in the Appendix. The code contains only the betatron motion useful for the study of BBU in linear accelerators, and the simulations have been performed with the idea that the instability arises in a time interval across transition which is less than the so called adiabatic time, given by [23]

$$
\tau_{a d}=\left(\frac{\pi \beta^{2} m_{0} c^{2} \gamma_{t}^{4}}{\dot{\gamma} \omega_{0}^{2} h e V\left|\cos \phi_{s}\right|}\right)^{1 / 3}
$$

with the relativistic $\beta$ factor about 1 at transition, $m_{0}$ the proton mass, $c$ the speed of light, $\gamma_{t}$ and $\dot{\gamma}$ the relativistic $\gamma$ at transition and its derivative with respect to time, $\omega_{0}$ the revolution angular frequency, $h$ the harmonic number, $e$ the electron charge, $V$ the rf peak voltage, and $\phi_{s}$ the synchronous phase. For the nTOF beam this time corresponds to about $1.8 \mathrm{~ms}$, while for the LHC beam it is about $1.4 \mathrm{~ms}$. Ignoring the longitudinal motion during this time, similar to a LINAC, BBU simulations can be performed. The result of the simulations can be compared with PyHEADTAIL. The observable is the growth of the transverse emittance in this interval using the same bunch parameters. For the BBU simulations, only the kickers' impedance has been considered and, for simplicity, it has

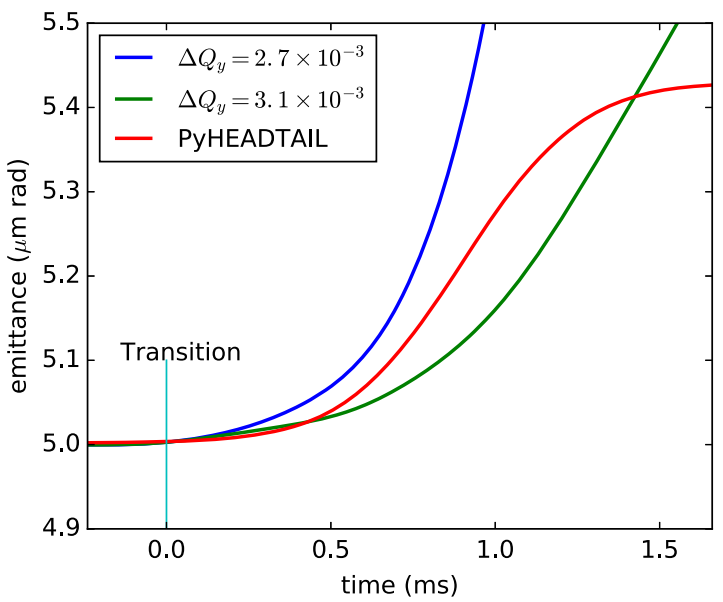

FIG. 18. Vertical normalized emittance vs time during transition crossing given by PyHEADTAIL and the BBU code for LHC (left side) and nTOF (right side) beams for two different tune spreads. 
been approximated by a single resonator, as shown in Fig. 17. The real part of the impedance, which is responsible for the instability, fits the kicker impedance quite well.

An important feature that needs to be used in the simulation is a small transverse tune spread induced by the quadrupolar (detuning) component of the impedance. PyHEADTAIL simulations have shown that this tune spread is very small, in the order of about $10^{-3}$, but this small value helps to reduce the instability growth. In Fig. 18 we show the normalized transverse emittance given by the BBU code as a function of time for the LHC beam with $27 \times 10^{10} \mathrm{ppb}$ (left side) and for the nTOF beam with $51 \times 10^{10} \mathrm{ppb}$ (right side) for two different tune spreads.

For PyHEADTAIL, the data is the same as those in Fig. 11 zoomed across transition energy. It is important to point out that even if the two codes use the same parameters, the beam dynamics for the BBU code is very different and simplified: no synchrotron motion, constant energy, constant bunch length, transverse impedance approximated by a single resonator, a small tune spread which should reproduce the effect of the quadrupolar impedance, and a completely different approach in taking into account the wakefield contribution. However, the final results are very similar, suggesting that the instability is indeed of BBU kind.

The BBU code also points out that the instability is very sensitive to the tune spread as discussed in the previous section.

\section{CONCLUSIONS AND OUTLOOK}

In this paper we presented the studies related to the instability occurring during the crossing of transition energy at the CERN Proton Synchrotron. We have performed several measurements obtaining the thresholds for different beam configurations and as a function of the longitudinal emittance. We have also measured the frequency spectrum characterizing the instability.

The developed PS impedance model has been used to simulate the instability with the PyHEADTAIL code.
Simulations showed that our present transverse impedance model is able to reproduce the instability characteristics, and that the kickers are the main source of this instability. The frequency spectra, under different conditions, are very similar to those obtained in the measurements. Good agreement of the instability threshold between simulations and measurements has been found for a large range of machine parameters, showing a discrepancy of about $30 \%$ only for large longitudinal emittances, with simulations being anyway more conservative and predicting a stronger instability.

Simulations also showed that this instability can be considered of a single bunch beam break-up kind, and it is very sensitive to any source of tune spread. We have also evaluated the mitigation effect due to the gamma jump scheme used for the nTOF and LHC beams, both in measurements and simulations. Finally, the influence of octupoles on the threshold has been demonstrated and measured.

\section{ACKNOWLEDGMENTS}

We acknowledge many helpful and stimulating discussions with N. Biancacci, H. Damerau, S. Gilardoni, S. Persichelli, and B. Salvant. The authors would like to thank K. Li, A. Oeftiger, and M. Schenk, for the support with PyHEADTAIL code, and all the PS-PSB Operation group for their help and support during the measurements. The work was performed in the framework of the PS-LIU project.

\section{APPENDIX: BEAM BREAK-UP SIMULATION CODE}

By following a formalism similar to that developed for the longitudinal case of Ref. [22], it is possible to obtain a transverse wakefield matrix, given by a single resonator, of the kind

$$
\mathbf{M}(z)=e^{-\frac{\omega_{r}}{2 Q} z / c}\left(\begin{array}{cc}
\cos \left(\omega_{n} z / c\right)+\frac{\omega_{r}}{2 Q \omega_{n}} \sin \left(\omega_{n} z / c\right) & \frac{1}{\omega_{n}} \sin \left(\omega_{n} z / c\right) \\
-\frac{\omega_{r}^{2}}{\omega_{n}} \sin \left(\omega_{n} z / c\right) & \cos \left(\omega_{n} z / c\right)-\frac{\omega_{r}}{2 Q \omega_{n}} \sin \left(\omega_{n} z / c\right)
\end{array}\right)
$$

with $\omega_{r}$ the resonant frequency of the resonator, $Q$ its quality factor, and $\omega_{n}$ the natural frequency. If we write the initial conditions for the induced wakefield voltage produced by a source charge $q$ with a transverse displacement $\Delta y_{1}$ as it is possible to obtain the induced voltage at the passage of the following charge at distance $z$ as

$$
\left[\begin{array}{c}
V(z) \\
\dot{V}(z)
\end{array}\right]=\mathbf{M}(z)\left[\begin{array}{c}
V(0) \\
\dot{V}(0)
\end{array}\right] .
$$

The second charge receives a transverse kick $\Delta \dot{y}=$ $\dot{V}(z) / E_{0}$ with $E_{0}$ the particle energy, and we can obtain the new initial conditions for the induced wakefield voltage as 


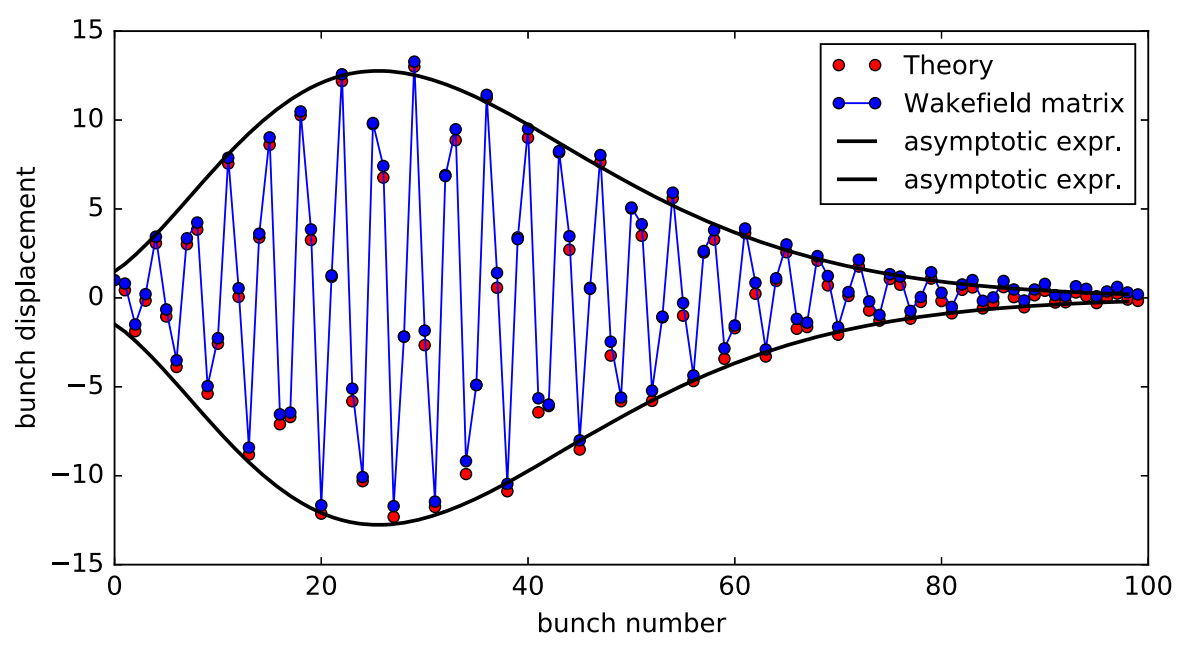

FIG. 19. Normalized displacements of the bunches for the multibunch BBU in transient regime.

$$
\left\{\begin{array}{l}
V(0)=V(z) \\
\dot{V}(0)=\dot{V}(z)+q \Delta y_{2} \frac{\omega_{r}}{2 Q} \omega_{n}
\end{array} .\right.
$$

These new conditions can be used back in Eq. (A3) to transport the induced wakefield up to the following charge and so forth. In this way, by ordering the particles from the head to the tail, it is possible to include the wakefield effects in beam dynamics simulations, both short and long range, with the only limit of approximating the machine coupling impedance by a sum of resonators. Each matrix can represent a resonator, and it is possible to study singlebunch and multibunch effects at the same time.

This method, with a different wakefield matrix, has been applied to the longitudinal beam dynamics of a circular accelerator in [22]. Here we apply the same method to the transverse beam dynamics of a LINAC to study the single bunch beam break-up. As example, in Fig. 19 we show a comparison of this method with the theory presented in [21] in the case of multi-bunch BBU, where each bunch is represented by a rigid macroparticle with identical charge. The machine and beam parameters are the same as those of Fig. 24 of the reference.

In the simulations performed in this paper, we considered a single bunch with a given longitudinal Gaussian distribution, and we transported the wakefield matrix from one macro-particle to the other within the same bunch.

[1] T. Risselada, Gamma transition jump scheme, CERN Accelerator School (CAS) Report No. CERN 85-19, 1985.

[2] E. Métral and D. Möhl, Transition crossing, Report No. CERN-2011-004, 2011, p. 59.

[3] M. Martini, CERN PS $\gamma_{t}$ jump Scheme, 1st mini-workshop on transition crossing FERMILAB-TM-1979, 1996.
[4] R. Cappi, E. Métral, and G. Métral, in Proceedings of the European Particle Accelerator Conference, Vienna, 2000 (EPS, Geneva, 2000), p. 1152.

[5] S. Aumon, B. Salvant, W. Bartmann, S. Gilardoni, E. Métral, G. Rumolo, and R. Steerenberg, in Proceedings of the 23rd Particle Accelerator Conference, Vancouver, Canada, 2009 (IEEE, Piscataway, NJ, 2009), p. 4649.

[6] S. Aumon, M. Delrieux, P. Freyermuth, S. Gilardoni, E. Métral, G. Rumolo, B. Salvant, and R. Steerenberg, in Proceedings of the International Particle Accelerator Conference, Kyoto, Japan (ICR, Kyoto, 2010), p. 2036.

[7] S. Aumon, H. Damerau, M. Delrieux, P. Freyermuth, S. Gilardoni, E. Métral, G. Rumolo, and B. Salvant, Proceedings of HB2010, Morschach, Switzerland (2010), p. 681, http://accelconf.web.cern.ch/AccelConf/HB2010/papers/ tho1d02.pdf.

[8] S. Hancock and E. Métral, Ghost bunches and blow-up losses with high-intensity beams, Report No. CERN PS/RF Note 2002-198, 2002.

[9] N. Wang, S. Aumon, N. Biancacci, G. Sterbini, M. Migliorati, and S. Persichelli, Proceedings of IPAC2016, Busan, Korea (2016), p. 1674-1677, http://accelconf.web .cern.ch/AccelConf/ipac2016/papers/tupor010.pdf.

[10] n-TOF collaboration, n-ToF Technical Design Report on Experimental Area, CERN Technical Report No. CERNINTC-2000-018, 2000.

[11] M. Migliorati, S. Persichelli, H. Damerau, S. Gilardoni, S. Hancock, and L. Palumbo, Beam-wall interaction in the CERN Proton Synchrotron for the LHC upgrade, Phys. Rev. ST Accel. Beams 16, 031001 (2013).

[12] S. Persichelli, M. Migliorati, N. Biancacci, S. Gilardoni, E. Metral, and B. Salvant, Transverse beam coupling impedance of the CERN Proton Synchrotron, Phys. Rev. Accel. Beams 19, 041001 (2016).

[13] https://github.com/PyCOMPLETE/PyHEADTAIL.

[14] S. Hancock, M. Lindroos, E. McIntosh, and M. Metcalf, Tomographic measurements of longitudinal phase space density, Comput. Phys. Commun. 118, 61 (1999). 
[15] R. Garoby, S. Hancock, and J. L. Vallet, in Proceedings of the European Particle Accelerator Conference, Vienna, 2000 (EPS, Geneva, 2000), p. 304.

[16] L. Palumbo, V. G. Vaccaro, and M. Zobov, CERN Report No. CERN 95-06, 1995, p. 331.

[17] A. Oeftiger and S. Hegglin, Proceedings of HB2016, Malmö, Sweden, MOPR025 (2016), http://accelconf.web .cern.ch/AccelConf/hb2016/papers/mopr025.pdf.

[18] M. Bassetti and G. A. Erskine, CERN Report No. CERNISR-TH/80-06, 1980.
[19] V. Kornilov, S. Aumon, S. Gilardoni, and P. Freyermuth, CERN Report No. CERN-ATS-Note-2013-038, MD, 2013.

[20] J. Gareyte, J. P. Koutchouk, and F. Ruggiero, LHC Project Report No. 91, 1997.

[21] A. Mosnier, CERN Report No. CERN 95-06, 2005, p. 481.

[22] M. Migliorati and L. Palumbo, Multibunch and multiparticle simulation code with an alternative approach to wakefield effects, Phys. Rev. ST Accel. Beams 18, 031001 (2015).

[23] S. Y. Lee, Accelerator Physics (World Scientific, Singapore, 2004). 